\title{
Performance of growth mixture models in the presence of time-varying covariates
}

\author{
Thierno M. O. Diallo ${ }^{1,2}$ • Alexandre J. S. Morin ${ }^{1} \cdot$ HuiZhong Lu$^{3}$
}

Published online: 31 October 2016

(C) Psychonomic Society, Inc. 2016

\begin{abstract}
Growth mixture modeling is often used to identify unobserved heterogeneity in populations. Despite the usefulness of growth mixture modeling in practice, little is known about the performance of this data analysis technique in the presence of time-varying covariates. In the present simulation study, we examined the impacts of five design factors: the proportion of the total variance of the outcome explained by the time-varying covariates, the number of time points, the error structure, the sample size, and the mixing ratio. More precisely, we examined the impact of these factors on the accuracy of parameter and standard error estimates, as well as on the class enumeration accuracy. Our results showed that the consistent Akaike information criterion (CAIC), the sample-size-adjusted CAIC (SCAIC), the Bayesian information criterion (BIC), and the integrated completed likelihood criterion (ICL-BIC) proved to be highly reliable indicators of the true number of latent classes in the data, across design conditions, and that the sample-size-adjusted BIC (SBIC) also proved quite accurate, especially in larger samples. In
\end{abstract}

Thierno M. O. Diallo and Alexandre J. S. Morin contributed equally to this work and both should be considered first authors.

Electronic supplementary material The online version of this article (doi:10.3758/s13428-016-0823-0) contains supplementary materials, which are available to authorized users.

Thierno M. O. Diallo

thdiallo.statmn@gmail.com

1 Institute for Positive Psychology and Education, Australian Catholic University, Strathfield campus, Locked Bag 2002, Strathfield, New South Wales 2135, Australia

2 Statistiques \& M.N., Sherbrooke, Canada

3 Computer Quebec/Canada, University of Sherbrooke, Sherbrooke, Canada contrast, the Akaike information criterion (AIC), the entropy, the normalized entropy criterion (NEC), and the classification likelihood criterion (CLC) proved to be unreliable indicators of the true number of latent classes in the data. Our results also showed that substantial biases in the parameter and standard error estimates tended to be associated with growth mixture models that included only four time points.

Keywords Growth mixture modeling · Time-varying covariates $\cdot$ Class enumeration $\cdot$ Bias $\cdot$ Coverage

Growth mixture models (GMMs; Muthén \& Shedden, 1999) are often used in applied research to explore qualitative differences in the growth trajectories observed within a sample (e.g., Morin, Maïano, Marsh, Nagengast, \& Janosz, 2013; Qureshi, \& Fang, 2011). The main advantage of GMMs is that they do not rely on the assumption that all individuals under study are drawn from a single population, which is the main limitation of more common latent growth models (LGMs; Bollen \& Curran, 2006). GMMs thus aim to explain longitudinal heterogeneity through the identification of unobserved subpopulations in the sample under study (Muthén, 2004; Muthén \& Shedden, 1999). In GMMs, population heterogeneity is captured by the inclusion of a categorical latent variable that identifies subgroups of individuals, typically referred to as latent profiles or latent classes. In fact, these latent profiles are akin to prototypes, and GMMs estimate the probabilities that each individual in the sample has of being a member of all of the extracted latent profiles (Muthén \& Shedden, 1999).

In GMMs, as in traditional LGMs, covariates can be included in the model to understand what influences the modeled inter- and intra-individual variability. Applications of GMMs often include time-invariant covariates (TICs), which are typically used to predict class membership as well 
as intraclass variability in the growth factors (e.g., Morin et al., 2011; Morin, Rodriguez, Fallu, Maïano, \& Janosz, 2012). Although less common, time-varying covariates (TVCs) can also be integrated to study their influence on the repeated outcome variable (Muthén, 2004; Peugh \& Fan, 2015).

\section{Class enumeration in GMMs}

Over and above the challenge of being able to recover true population parameters that is common to all statistical models, mixture models (including GMMs) are complicated by the critical challenge of extracting the correct number of latent classes in the data. Currently, a combination of statistical tests and indices, and conformity with substantive theory, are used to determine the number of classes (class enumeration) in the context of mixture modeling in general, and GMMs in particular (e.g., Li \& Hser, 2011; Muthén, 2003; Tofighi \& Enders, 2007). Among the most commonly used indicators are the information criteria (ICs), as well as some entropy-based criteria. In the IC family, five indicators are typically used: the Bayesian information criterion (BIC; Schwarz, 1978), a sample-size-adjusted BIC (SBIC; Sclove, 1987), the Akaike information criterion (AIC; Akaike, 1987), the consistent AIC (CAIC; Bozdogan, 1987), and a sample-size-adjusted CAIC (SCAIC; Tofighi \& Enders, 2007). Similarly, four common entropy-based indicators exist, although these indicators have not been typically used in the GMM class enumeration process: the entropy (Lubke \& Muthén, 2007; Ramaswamy, DeSarbo, Reibstein, \& Robinson, 1993), the normalized entropy criterion (NEC; Celeux \& Soromenho, 1996), the classification likelihood criterion (CLC; Biernacki \& Govaert, 1997; McLachlan $\&$ Peel, 2000), and the integrated completed likelihood criterion (a BIC indicator adjusted for entropy [ICL-BIC]; Biernacki, Celeux, \& Govaert, 2000; McLachlan \& Peel, 2000).

\section{Growth mixture models with TVCs}

Although multiple studies have investigated the performance of these indicators in the class enumeration process of a variety of mixture models (we review these studies in Appendix 1 of the online supplements), these studies remain limited in some areas. In particular, one form of complexity that has been considered in only one GMM study (Peugh \& Fan, 2015) is the inclusion of TVCs. In this study, Peugh and Fan examined the class enumeration accuracy of GMMs when TICs, TVCs, and one outcome variable were included in both the population model and the class enumeration process. The accuracy of most of the previously listed indicators was investigated (as well as a few additional ones), as a function of sample size $(500,1,500$, and 3,000), degree of class separation (with Mahalanobis distances between the classes equal to 1 and 2), mixing ratio $(33 \%-33 \%-33 \%, 50 \%-33 \%-17 \%$, and $65 \%-$ $25 \%-10 \%$ ), and the percentage of variance explained by the TICs and the TVCs $(10 \%$ and $25 \%)$. With a sample size of 500 , most indicators had low levels of class enumeration accuracy, with strong underextraction tendencies for the CAIC, BIC, and ICL-BIC. In fact, GMM including TICs, TVCs, and the outcome performed poorly with sample sizes lower than 3,000 . In contrast, these models had higher class enumeration accuracy in conditions of sample size equal to 3,000 , high class separation, equal mixing ratios, and $25 \%$ variance explained by the covariates (TICs and TVCs). In general, across conditions, the sample-size-adjusted versions of the ICs were more accurate than the non-sample-size-adjusted versions. However, a key limitation of this study was that all population and estimated models included all of the covariates (TICs, TVCs, and outcome), making it impossible to determine which part of the observed results was due to the presence of the TVCs, relative to the TICs and to the outcome variable.

Furthermore, only three prior studies (Kim, 2012; Tofighi \& Enders, 2007; Tolvanen, 2008) investigated the impact of a limited number of measurement points on the performance of GMM - an element that may prove critical as far as TVCs are concerned and that was ignored by Peugh and Fan (2015). Furthermore, one of these studies (Tofighi \& Enders, 2007) relied on a partial factorial design in which the normative condition involved four measurement points, limiting the possible conclusions in this regard.

Another question that remains unanswered is related to the impact of the residual structure on the class enumeration accuracy of GMMs (e.g., Enders \& Tofighi, 2008; Tolvanen, 2008) - another design factor that may prove critical in the presence of TVCs and was not investigated by Peugh and Fan (2015). Indeed, the influence of TVCs on the repeated measures is directly, and inversely, related to the size of the residuals (i.e., or directly related to the $R^{2}$ of the repeated measures), and is also intimately related to the structure of these residuals (homoscedastic, heteroscedastic, class-invariant, class-varying; see the online supplements). This is an important limitation given that the $R^{2}$ of the repeated measures (and inversely the size and structure of the time-specific residuals) reflects the effect size of the model, which represent a key factor in power analyses (e.g., Cohen, 1988) and an important determinant of power in LGM (Diallo \& Morin, 2015; Diallo, Morin, \& Parker, 2014). In the only study to date that considered this design factor for GMM without covariates, Tolvanen (2008) reported that the class enumeration was more accurate when the $R^{2}$ of the repeated measures was higher, a condition that also reduced estimation biases. It would be interesting to see whether these results hold in the presence of TVCs, and the likely impact of the proportion of the global $R^{2}$ of the repeated measures that is due to the TVCs. Furthermore, although Tolvanen (2008) considered the effects of the $R^{2}$ conditions, he did not consider the fact that the residual structure could be 
either heteroscedastic or homoscedastic across time points. The homoscedasticity of the residuals is a common assumption in LGM (e.g., Cheong, 2011; Fan \& Fan, 2005), which has been shown to result in biases in GMM when the population model is heteroscedastic (Enders \& Tofighi, 2008).

Hence, the purpose of this study was to evaluate the effects of multiple design conditions (the proportion of the total variance of the outcome- $R^{2}$ - explained by the TVCs, the number of time points, the residual structure, the sample size, and the mixing ratio) on the parameter recovery of GMMs with TVCs. The performance of the common ICs in terms of class enumeration accuracy under the various design conditions considered here will also be examined. The mathematical expressions of the indicators considered in the present study are presented in Table $\mathrm{S} 1$ of the online supplements.

\section{The specification of GMMs with TVCs}

LGMs with TVCs LGMs (Bollen \& Curran, 2006; Meredith \& Tisak, 1990) are used to analyze multiwave longitudinal data when population homogeneity is assumed for the estimated population parameters and the global shape of the growth trajectories. In LGMs, TVCs are directly included in the model as predictors of the repeated measures (Bollen \& Curran, 2006; Muthén \& Curran, 1997). In the structural equation modeling approach to LGMs with TVCs that forms the basis of the GMM approach considered later, the effects of TVCs may vary across time but are specified as constant across individuals. Assuming a linear model for the repeated measures, for individual $i$ at time $t(t=1,2, \ldots, T)$, the withinperson longitudinal LGM with one TVC is represented as:

$y_{i t}=\alpha_{i}+\beta_{i} \lambda_{t}+\gamma_{t} w_{i t}+\varepsilon_{i t}$,

where $y_{i t}$ is the repeated measure outcome variable, $\lambda_{t}$ is the time score, $\alpha_{i}$ is the random intercept, $\beta_{i}$ is the random linear slope, $\gamma_{t}$ is the slope of the repeated measure on the TVC $w_{i t}$ (allowed to vary freely across time points), and $\varepsilon_{i t} \sim N\left(0, \theta_{t}\right)$ is the normally distributed residual for $y_{i t}$. Growth is represented by imposing constraints on the time scores $\left(\lambda_{t}\right)$ reflecting the passage of time. LGMs generally assume that the growth factors, the TVC, and the time-specific residuals are mutually independent and multivariate normal. The betweenperson LGM model with a TVC is specified as:

$\alpha_{i}=\mu_{\alpha}+\zeta_{\alpha i}$

$\beta_{i}=\mu_{\beta}+\zeta_{\beta_{i}}$

where $\mu_{\alpha}, \mu_{\beta}$ are the means of the growth factors, and $\zeta_{\alpha i}$ and $\zeta_{\beta i}$ are the multivariate normal residuals of growth factors with a zero mean vector, $\operatorname{Var}(\alpha)=\operatorname{Var}\left(\zeta_{\alpha i}\right)=\psi_{\alpha y \alpha y}, \operatorname{Var}(\beta)=$ $\operatorname{Var}\left(\zeta_{\beta i}\right)=\psi_{\beta y \beta y}$ and $\operatorname{Cov}(\alpha, \beta)=\operatorname{Cov}\left(\zeta_{\alpha i}, \zeta_{\beta i}\right)=\psi_{\alpha y \beta y}$

GMMs with TVCs As we noted, GMMs relax the assumption of population homogeneity by relying on a finite mixture approach for handling heterogeneity in growth trajectories. A categorical latent variable is used to model heterogeneity in the growth trajectories, so that each latent class is characterized by its own LGM. In GMMs with a TVC, under multivariate normality and independency assumptions, the equations for the within- and between-persons models are, respectively,

$y_{i t}=\sum_{k=1}^{K} p_{i}^{(k)}\left[\alpha_{i}^{(k)}+\lambda_{t} \beta_{i}^{(k)}+\gamma_{t} w_{i t}^{(k)}+\varepsilon_{i t}\right]$,

and

$$
\begin{aligned}
\alpha_{i}^{(k)} & =\mu_{\alpha}^{(k)}+\zeta_{\alpha i}^{(k)}, \beta_{i}^{(k)} \\
& =\mu_{\beta}^{(k)}+\zeta_{\beta i}^{(k)}, \varepsilon_{i t} \sim N\left(0, \theta_{t}\right), \quad \operatorname{Var}\left(\alpha^{(k)}\right) \\
& =\psi_{\alpha y \alpha y}^{(k)}, \quad \operatorname{Var}\left(\beta^{(k)}\right) \\
& =\psi_{\beta y \beta y}^{(k)}, \quad \text { and } \operatorname{Cov}\left(\alpha^{(k)}, \beta^{(k)}\right)=\psi_{\alpha y \beta y}^{(k)} .
\end{aligned}
$$

The parameters are the same as in the LGM, and a superscript $(k)$ is appended to each parameter to indicate that it is allowed to vary across latent classes $(k=1,2, \ldots, K)$. The set of mixing proportion $p_{i}^{(k)} \mathrm{s}$ defines the probability that an individual belongs to the $k$ class with all $p_{i}^{(k)} \geq 0$ and $\sum_{k=1}^{K} p_{i}^{(k)}=1$. It is noteworthy that, in this study, $\lambda_{t}, \gamma_{t}$, and $\varepsilon_{i t}$ are specified as invariant across classes, but allowed to vary across time points.

As we noted above, our objective in the present simulation study was to investigate the parameter recovery and class enumeration accuracy of GMMs with TVCs as a function of a variety of design conditions, rather than to provide a detailed introduction to the practical estimation and interpretation of these models. However, interested readers are encouraged to consult Liang, Xu, Bennett, Ye, and Quiñones (2009) for an applied research example using GMMs with TVCs, Morin (2016; Morin \& Wang, 2016) for a nontechnical introduction to the estimation of a variety of mixture models, including GMMs, and to Ram and Grimm (2009; see also Grimm, Ram, \& Estabrook, 2010) for more advanced introductions to GMMs. In Appendix 3 of the online supplements, we also provide an annotated Mplus setup for one of the simulation runs. Other simulation conditions can be specified by changing certain parameter values in the model simulation section of the setup, as we describe in the design of the simulation. A similar input setup for the practical estimation of the same model with real data is also provided, with a variety of optional settings. 


\section{Method}

A linear model with two classes and one TVC was used as the population model for this simulation study. A challenge in simulation studies is to put a limit on the design conditions that are considered, in order to keep the studies within reasonable computational boundaries. On this basis, and in accordance with similar decisions made in previous simulation studies (Li \& Hser, 2011; Liu \& Hancock, 2014; Nylund, Asparouhov, \& Muthén, 2007; Tolvanen, 2008), we decided to rely on a two-class population model. The population parameters are provided in Table 1. These parameter values result in an intercept mean difference of three standard deviations between the two classes, corresponding to a situation of high class separation according to Tofighi and Enders (2007) and $\operatorname{Kim}(2012,2014)$.

\section{Manipulated factors}

On the basis of their likely impact on the class enumeration and parameter recovery of GMMs, five factors were manipulated in this study: the proportion of the total variance of the outcome $\left(R^{2}\right)$ explained by the time-varying covariate at each time point, the number of time points, the residual structure, the sample size, and the mixing proportion. Previous studies had already shown that sample size (e.g., Enders \& Tofighi, 2008; Li, \& Hser, 2011; Liu \& Hancock, 2014; Lubke \& Muthén, 2007; Peugh \& Fan, 2012, 2015; Tofighi \& Enders, 2007) and having an equal mixing ratio (e.g., Enders \& Tofighi, 2008; Nylund et al. 2007; Peugh \& Fan, 2012, 2015; Tofighi \& Enders, 2007) had a positive impact on the class enumeration and parameter recovery of GMMs. However, this study was the first to systematically investigate the impact of the residual design factors.

Sample sizes Following previous studies (Li \& Hser, 2011; Nylund et al., 2007; Yang, 2006) and typical GMM applications (e.g., Fanti \& Henrich, 2010; Schaeffer, Petras, Ialongo, Poduska, \& Kellam, 2003; Sterba, Prinstein, \& Cox, 2007), we considered four conditions, corresponding to small, medium, large, and very large sample sizes: $250,500,1,000$, and 2,000.

Number of measurement points Three different conditions were considered regarding the number of measurement points: four, six, and eight. Four measurement points are often used in simulation studies of GMMs (Enders \& Tofighi, 2008; Liu \& Hancock, 2014; Nylund et al., 2007; Peugh \& Fan, 2015;

Table 1 Population parameters for the simulation study

\begin{tabular}{|c|c|c|}
\hline Parameter & Class 1 & Class 2 \\
\hline \multicolumn{3}{|l|}{ Growth factors means } \\
\hline Intercept mean, $\mu_{\alpha}$ & 30 & 15 \\
\hline Linear slope mean, $\mu_{\beta}$ & 2.91 & 0.39 \\
\hline \multicolumn{3}{|c|}{ Growth factors variances and covariances } \\
\hline Intercept variance, $\psi_{\alpha y \alpha y}$ & 5 & 5 \\
\hline Slope variance, $\psi_{\beta y \beta y}$ & 0.50 & 0.50 \\
\hline Intercept/slope covariance, $\psi_{\alpha y \beta y}$ & 0.03 & 0.03 \\
\hline \multicolumn{3}{|l|}{ Slope of the TVC $\gamma_{t}$} \\
\hline \multicolumn{3}{|l|}{ Homoscedastic condition } \\
\hline \multicolumn{3}{|l|}{$2 \%$ of explained variance } \\
\hline $\begin{array}{l}\gamma_{1} ; \gamma_{2} ; \gamma_{3} ; \gamma_{4} ; \gamma_{5} ; \gamma_{6} ; \gamma_{7} ; \gamma_{8} \\
7 \% \text { of explained variance }\end{array}$ & $0.88 ; 0.88 ; 0.90 ; 0.93 ; 0.98 ; 1.03 ; 1.09 ; 1.15$ & $0.88 ; 0.88 ; 0.90 ; 0.93 ; 0.98 ; 1.03 ; 1.09 ; 1.15$ \\
\hline $\begin{array}{l}\gamma_{1} ; \gamma_{2} ; \gamma_{3} ; \gamma_{4} ; \gamma_{5} ; \gamma_{6} ; \gamma_{7} ; \gamma_{8} \\
\text { Heteroscedastic condition } \\
2 \% \text { of explained variance }\end{array}$ & $1.68 ; 1.70 ; 1.73 ; 1.79 ; 1.87 ; 1.97 ; 2.09 ; 2.21$ & $1.68 ; 1.70 ; 1.73 ; 1.79 ; 1.87 ; 1.97 ; 2.09 ; 2.21$ \\
\hline $\begin{array}{l}\gamma_{1} ; \gamma_{2} ; \gamma_{3} ; \gamma_{4} ; \gamma_{5} ; \gamma_{6} ; \gamma_{7} ; \gamma_{8} \\
7 \% \text { of explained variance }\end{array}$ & $0.86 ; 1.02 ; 1.01 ; 0.98 ; 0.96 ; 0.99 ; 1.02 ; 1.11$ & $0.86 ; 1.02 ; 1.01 ; 0.98 ; 0.96 ; 0.99 ; 1.02 ; 1.11$ \\
\hline $\begin{array}{l}\gamma_{1} ; \gamma_{2} ; \gamma_{3} ; \gamma_{4} ; \gamma_{5} ; \gamma_{6} ; \gamma_{7} ; \gamma_{8} \\
\text { Residual variances }\end{array}$ & $1.64 ; 1.95 ; 1.94 ; 1.89 ; 1.85 ; 1.91 ; 1.96 ; 2.12$ & $1.64 ; 1.95 ; 1.94 ; 1.89 ; 1.85 ; 1.91 ; 1.96 ; 2.12$ \\
\hline Homoscedastic condition & & \\
\hline $\begin{array}{l}\theta_{1}=\theta_{2}=\theta_{3}=\theta_{4}=\theta_{5}=\theta_{6}=\theta_{7}=\theta_{8} \\
\text { Heteroscedastic condition }\end{array}$ & 21.81 & 21.81 \\
\hline$\theta_{1} ; \theta_{2} ; \theta_{3} ; \theta_{4} ; \theta_{5} ; \theta_{6} ; \theta_{7} ; \theta_{8}$ & $21.02 ; 34.12 ; 31.61 ; 26.40 ; 20.72 ; 18.55 ; 14.87 ; 16.49$ & $21.02 ; 34.12 ; 31.61 ; 26.40 ; 20.72 ; 18.55 ; 14.87 ; 16.49$ \\
\hline
\end{tabular}


Tofighi \& Enders, 2007; Tolvanen, 2008). The other two numbers were selected to reflect moderate and elevated numbers of measurement occasions that are commonly seen in applied GMM research (e.g., Muthén et al., 2002; Sterba, Prinstein, \& Cox, 2007).

Mixing ratio Following previous research showing this factor to be critical in the ability to recover the true number of classes present in the data (e.g., Liu \& Hancock, 2014; Nylund et al., 2007; Tofighi \& Enders, 2007), two different conditions were considered in this study. In the first condition, the sizes of the two latent classes forming the population model were specified as equal to one another, resulting in a mixing ratio of .50/.50. In the second condition, one latent class was simulated as being smaller than the other one, resulting in a mixing ratio of .30/.70.

Residual structure As previously noted, the impact of the residual structure on the class enumeration accuracy and parameter recovery of GMM is currently unknown. Given that the residual structure is intimately related to the effect size of the model, it appears important to consider this factor as a design condition. In this study, the data were simulated on the basis of two different residual structures. In the first condition, the residuals were specified as equal across time points (i.e., homoscedastic) and latent classes. In the second condition, the time-specific residuals were specified as different across time points (i.e., heteroscedastic), but equal across latent classes.

$\mathbf{R}^{2}$ associated with the TVC Following Muthén and Muthén (2002) in the context of LGMs with TICs, and Peugh and Fan (2015) in the context of GMMs with TVCs, the proportion of the variance of the outcome explained by the TVC at each time points was varied. The values of the slopes of the simulated data were chosen so that the TVC accounted for $2 \%$ and $7 \%$ of the variance in the repeated measures at each time point. These proportions of explained variance roughly correspond to small and medium effect sizes (Cohen, 1988). Although somewhat arbitrary, these values were chosen to replicate those selected by Tofighi and Enders (2007) in their study of GMMs with TICs. The $R^{2}$ of the repeated measures reflect the sums of the variance explained in the repeated measures by the growth trajectory factors, and by the TVC. Given the population parameters selected in this study and the manipulation of the proportion of explained variance in the repeated measures due to the TVC to be either $2 \%$ or $7 \%$, the global $R^{2}$ of the repeated measures ranged from small (.32) to large (.73) across conditions. The exact formulas allowing for the precise calculation of $R^{2}$ in the context of GMMs with TVCs are provided in Appendix 3 of the online supplements.

\section{Data generation}

In summary, we implement five fully crossed design conditions, producing a total of 96 unique cells (4 sample sizes $\times 3$ measurement points $\times 2$ mixing ratio $\times 2 R^{2}$ values $\times 2$ error structures $=96$ ). All models were simulated and estimated using Mplus 7.0's Monte Carlo capabilities (Muthén \& Muthén, 2012). A total of 1,000 replications were generated within each analyzed cell, using a multivariate normal distribution within each class. To verify the ability of the models to identify the correct number of latent classes in the data $(k=2$ in all conditions considered here), models including one to four latent classes were systematically evaluated for all of the design conditions. Apart from misspecifications related to the number of latent classes, all of these models were otherwise specified correctly. For two classes or more, we used 400 sets of random starting values and 40 iterations for each set of the random starting values. The 20 solutions with the highest likelihood values were selected and iterated in the final stage. In the model evaluation, models that did not converge, or that converged on an improper solution, were discarded, and all results presented here are based on converged proper solutions. ${ }^{1}$ This simulation study was made possible through the use of Compute Canada's high-performance computing facilities (https://computecanada.ca/).

\section{Outcomes}

The main outcomes are the ability of the ICs (BIC, SBIC, AIC, CAIC, and SCAIC) and entropy-based indicators (entropy, CLC, NEC, and ICL-BIC) to correctly identify the presence of two latent classes in the data, as well as the accuracy of parameter estimates in the correctly specified twoclass models. For the class enumeration accuracy, we examined the proportion of replications in which each of the indicators correctly identified the two-class model as the correct solution. For the five ICs, as well as the CLC, NEC, and ICL$\mathrm{BIC}$, the model associated with the lowest value among the

\footnotetext{
${ }^{1}$ Following the standard practice in mixture modeling simulation studies (e.g., Bauer \& Curran, 2003; McLachlan \& Peel, 2000), a replication was labeled nonconvergent when that replication failed to converge on a solution with any of the sets of the starting values that were used. A convergent solution was labeled as inadmissible or improper when it included a parameter estimates that fell outside of its permissible boundaries (i.e., negative variances, correlations greater than 1 or smaller than -1 ). Because inadmissible solutions are rarely interpreted (Chen, Bollen, Paxton, Curran, \& Kirby, 2001) they were excluded from the results reported here.
} 
Table 2 Eta squared of the ANOVA of the indicators in selecting the true two-class model as a function of the number of time points, sample sizes, error structures, mixing ratio, and effect sizes

\begin{tabular}{|c|c|c|c|c|c|c|c|c|}
\hline Factors & AIC & CAIC & SCAIC & $\mathrm{BIC}$ & SBIC & Entropy & NEC & ICL-BIC \\
\hline Sample Sizes (A) & 0.18 & 0.05 & 0.89 & 0.09 & 0.96 & 0.07 & 0.65 & 0.16 \\
\hline Time Points (B) & 0.26 & 0.05 & 0.03 & 0.02 & 0.01 & 0.88 & 0.27 & 0.01 \\
\hline Mixing Ratio (C) & 0.02 & 0 & 0 & 0.03 & 0 & 0 & 0 & 0.01 \\
\hline Residual (D) & 0.03 & 0 & 0 & 0 & 0 & 0.01 & 0 & 0 \\
\hline TVC-R ${ }^{2}(\mathrm{E})$ & 0 & 0 & 0 & 0 & 0 & 0 & 0 & 0 \\
\hline $\mathrm{A} * \mathrm{~B}$ & 0.31 & 0.15 & 0.03 & 0.31 & 0.02 & 0.01 & 0.07 & 0.27 \\
\hline $\mathrm{A}^{*} \mathrm{C}$ & 0 & 0.05 & 0.01 & 0.09 & 0 & 0 & 0 & 0.07 \\
\hline$A * D$ & 0.01 & 0.05 & 0 & 0.02 & 0 & 0 & 0 & 0.01 \\
\hline$A * E$ & 0 & 0 & 0 & 0 & 0 & 0 & 0 & 0 \\
\hline $\mathrm{B} * \mathrm{C}$ & 0.07 & 0.05 & 0.01 & 0.01 & 0 & 0 & 0 & 0.01 \\
\hline$B * D$ & 0 & 0.05 & 0.01 & 0 & 0 & 0.01 & 0 & 0 \\
\hline$B * E$ & 0 & 0 & 0 & 0 & 0 & 0 & 0 & 0 \\
\hline$C * D$ & 0 & 0 & 0 & 0.01 & 0 & 0 & 0 & 0.01 \\
\hline$C^{*} \mathrm{E}$ & 0 & 0 & 0 & 0 & 0 & 0 & 0 & 0 \\
\hline$D^{*} E$ & 0 & 0 & 0 & 0 & 0 & 0 & 0 & 0 \\
\hline
\end{tabular}

$\mathrm{AIC}=$ Akaike's information criterion; $\mathrm{CAIC}=$ consistent $\mathrm{AIC} ; \mathrm{SCAIC}=$ sample size adjusted CAIC; $\mathrm{BIC}=$ Bayesian information criterion; $\mathrm{SBIC}=$ sample size adjusted BIC; NEC = normalized entropy criterion; ICL-BIC $=$ integrated completed likelihood criterion; $\mathrm{C}=$ class.

four competing models is considered the best-fitting model. For the entropy indicator, the model with the highest value indicates the best-fitting model. The accuracy of the parameter estimates is evaluated with the relative percentage bias ${ }^{2}$ (RPB) of those estimates and of their corresponding standard errors, and the coverage of the $95 \%$ confidence interval. The RPB is defined as the percentage of underestimation or overestimation of a parameter estimate relative to its true population value (e.g., Hoogland \& Boomsma, 1998; Kaplan, 1989). The RPBs of the standard errors evaluate whether the standard errors of the parameter estimates accurately reflect the sampling variation in the estimation of the parameter. The $95 \%$ coverage indicates the percentage of replications in which the true value of the parameter falls within the estimated $95 \%$ confidence interval.

A series of analyses of variance (ANOVAs) were conducted to examine the impacts of all the design factors on the ability of each indicator to correctly retain the true number of latent classes present in the data, as well as on the RPBs of the parameter estimates and their corresponding standard errors. Since 96,000 unique solutions are considered here (96 conditions $\times 1,000$ replications), extremely small biases can be detected at a standard significance level of $p \leq .05$. Indeed, because statistical significance tests are highly sensitive to sample size, they tend to be less informative in the comparison of results obtained from different design conditions in the

\footnotetext{
2 The relative percentage bias of parameter estimate is defined as $R P B(\hat{\theta})$ $=(\hat{\theta}-\theta) / \theta * 100 \%$, where $\hat{\theta}$ and $\theta$ are the average parameter estimate and the true population value, respectively. For the relative percentage bias of standard error of the parameter estimates, $\operatorname{RPB}(\operatorname{se}(\hat{\theta}))=$ $[s e(\hat{\theta})-s d(\theta)] / s d(\theta) * 100 \%$, where $s e(\hat{\theta})$ and $s d(\theta)$ are the averages of the estimated standard errors and the standard deviations of the parameter estimates, respectively.
}

context of simulation studies, in which one only has to increase the number of replications (i.e., the "sample" size associated with the design condition) to reach significance. For this reason, the proportion of variation in the outcomes $\left(\eta^{2}\right)$ that is accounted for by each of the design factors and their two-way interactions is used as an effect size indicator. Following Cohen (1988), we used $\eta^{2}=.01, .06$, and .14 to define small, moderate, and large effect sizes, respectively. $\eta^{2}$ $\geq .06$ was adopted for filtering out the effects that were trivial in magnitude as we evaluated the impact of each design factor. Although we focus on the main conclusions, extensive results tables are available in the online supplements.

\section{Results}

\section{Class enumeration}

Table 2 provides the ANOVA results for the effects of all design conditions on the ability of each indicator to correctly retain the true number of latent classes present in the data. For illustration purposes, the detailed results for all indicators, associated with the four time point condition, are reported in Tables 3, 4, 5 and 6. Tables S2-S9 of the online supplements provide the class enumeration accuracy associated with six and eight time points. The ANOVA could not be computed for the CLC because this indicator never selected the two-class solution, and thus should not be considered a reliable indicator of class enumeration. Although slightly better than that of the CLC, the performance of the NEC was equally poor, correctly identifying the two-class solution only $16.21 \%$ of the time across conditions. For this reason, these indicators will not be discussed further. The results show that all indicators are 
Table 3 Class enumeration accuracy of the indicators as a function of sample size, for a mixing ratio of $050 / 0.50$ and four time points

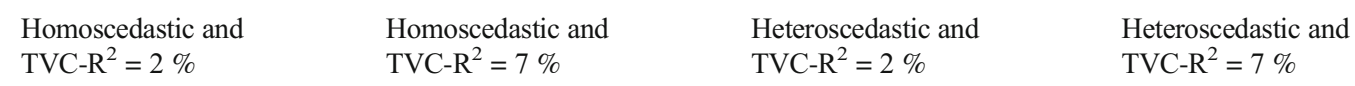

\begin{tabular}{|c|c|c|c|c|c|c|c|c|c|c|c|c|c|c|c|c|c|}
\hline \multirow{2}{*}{$\begin{array}{l}\# \text { of Classes } \\
N=250\end{array}$} & & 1 & 2 & 3 & 4 & 1 & 2 & 3 & 4 & 1 & 2 & 3 & 4 & 1 & 2 & 3 & 4 \\
\hline & $\mathrm{AIC}$ & 0 & 74.6 & 19.1 & 6.3 & 0 & 74.6 & 19.1 & 6.3 & 0 & 69.6 & 22.2 & 8.2 & 0 & 69.6 & 22.2 & 8.2 \\
\hline & CAIC & 0 & 100 & 0 & 0 & 0 & 100 & 0 & 0 & 0 & 100 & 0 & 0 & 0 & 100 & 0 & 0 \\
\hline & SCAIC & 0 & 96.3 & 3.7 & 0 & 0 & 96.3 & 3.7 & 0 & 0 & 94.1 & 5.9 & 0 & 0 & 94.1 & 5.9 & 0 \\
\hline & BIC & 0 & 100 & 0 & 0 & 0 & 100 & 0 & 0 & 0 & 100 & 0 & 0 & 0 & 100 & 0 & 0 \\
\hline & SBIC & 0 & 83.1 & 13.2 & 3.7 & 0 & 83.1 & 13.2 & 3.7 & 0 & 79.2 & 17.8 & 3 & 0 & 79.2 & 17.8 & 3 \\
\hline & Entropy & 0 & 63.5 & 28.6 & 7.9 & 0 & 63.5 & 28.6 & 7.9 & 0 & 53.3 & 34.1 & 12.6 & 0 & 53.3 & 34.1 & 12.6 \\
\hline & CLC & 0 & 0 & 0 & 100 & 0 & 0 & 0 & 100 & 0 & 0 & 0.7 & 99.3 & 0 & 0 & 0.7 & 99.3 \\
\hline & NEC & 0 & 3.7 & 13.2 & 83.1 & 0 & 3.7 & 13.2 & 83.1 & 0 & 4.4 & 10.4 & 85.2 & 0 & 4.4 & 10.4 & 85.2 \\
\hline & ICL-BIC & 0 & 100 & 0 & 0 & 0 & 100 & 0 & 0 & 0 & 100 & 0 & 0 & 0 & 100 & 0 & 0 \\
\hline$N=500$ & $\mathrm{AIC}$ & 0 & 76.8 & 18.5 & 4.7 & 0 & 76.8 & 18.5 & 4.7 & 0 & 74.8 & 22 & 3.2 & 0 & 74.8 & 22 & 3.2 \\
\hline & CAIC & 0 & 100 & 0 & 0 & 0 & 100 & 0 & 0 & 0 & 100 & 0 & 0 & 0 & 100 & 0 & 0 \\
\hline & SCAIC & 0 & 98.7 & 1.3 & 0 & 0 & 98.7 & 1.3 & 0 & 0 & 98.2 & 1.8 & 0 & 0 & 98.2 & 1.8 & 0 \\
\hline & $\mathrm{BIC}$ & 0 & 100 & 0 & 0 & 0 & 100 & 0 & 0 & 0 & 100 & 0 & 0 & 0 & 100 & 0 & 0 \\
\hline & SBIC & 0 & 94.6 & 5.4 & 0 & 0 & 94.6 & 5.4 & 0 & 0 & 92.7 & 7.3 & 0 & 0 & 92.7 & 7.3 & 0 \\
\hline & Entropy & 0 & 62.3 & 32 & 5.7 & 0 & 62.3 & 32 & 5.7 & 0 & 54.6 & 38.1 & 7.3 & 0 & 54.6 & 38.1 & 7.3 \\
\hline & CLC & 0 & 0 & 0.3 & 99.7 & 0 & 0 & 0.3 & 99.7 & 0 & 0 & 1.4 & 98.6 & 0 & 0 & 1.4 & 98.6 \\
\hline & NEC & 0 & 18.5 & 24.2 & 57.3 & 0 & 18.5 & 24.2 & 57.3 & 0 & 27.5 & 22 & 50.5 & 0 & 27.5 & 22 & 50.5 \\
\hline & ICL-BIC & 0 & 100 & 0 & 0 & 0 & 100 & 0 & 0 & 0 & 100 & 0 & 0 & 0 & 100 & 0 & 0 \\
\hline
\end{tabular}

TVC $=$ time-varying covariate $;$ AIC $=$ Akaike's information criterion $;$ CAIC $=$ consistent AIC $;$ SCAIC $=$ sample size adjusted CAIC BIC $=$ Bayesian information criterion; SBIC = sample size adjusted BIC; NEC = normalized entropy criterion; ICL-BIC = integrated completed likelihood criterion.

significantly influenced by the sample size and the number of time points in correctly selecting the true number of classes in the data. However, although the mixing ratio, residual structure, and $R^{2}$ associated with the TVC also influenced the accuracy of some indicators, these effects generally remained trivial. A few two-way interactions were also significant.
Main effect of sample size The effects of sample size differed according to the indicator considered, ranging from moderate (for BIC $\left[\eta^{2}=.09\right]$ and entropy $\left[\eta^{2}=.07\right]$ ) to large (for SBIC $\left[\eta^{2}=.96\right]$, SCAIC $\left[\eta^{2}=.89\right]$, AIC $\left[\eta^{2}=\right.$ $0.18]$, and ICL-BIC $\left.\left[\eta^{2}=.16\right]\right)$. Among the ICs, the AIC did not perform as well as the other indicators, with a rate of correct identification of the two-class solution ranging

Table 4 Class enumeration accuracy of the indicators as a function of sample size, for a mixing ratio of $0.50 / 0.50$ and four time points

\begin{tabular}{|c|c|c|c|c|c|c|c|c|c|c|c|c|c|c|c|c|c|}
\hline \multirow[b]{2}{*}{ \# of Classes } & & \multicolumn{4}{|c|}{$\begin{array}{l}\text { Homoscedastic and } \\
\text { TVC- } \mathrm{R}^{2}=2 \%\end{array}$} & \multicolumn{4}{|c|}{$\begin{array}{l}\text { Homoscedastic and } \\
\text { TVC- }^{2}=7 \%\end{array}$} & \multicolumn{4}{|c|}{$\begin{array}{l}\text { Heteroscedastic and } \\
\text { TVC- }^{2}=2 \%\end{array}$} & \multicolumn{4}{|c|}{$\begin{array}{l}\text { Heteroscedastic and } \\
\text { TVC- } \mathrm{R}^{2}=7 \%\end{array}$} \\
\hline & & 1 & 2 & 3 & 4 & 1 & 2 & 3 & 4 & 1 & 2 & 3 & 4 & 1 & 2 & 3 & 4 \\
\hline \multirow[t]{9}{*}{$N=1,000$} & AIC & 0 & 77.5 & 18.9 & 3.6 & 0 & 77.5 & 18.9 & 3.6 & 0 & 78.6 & 18 & 3.4 & 0 & 78.6 & 18 & 3.4 \\
\hline & CAIC & 0 & 100 & 0 & 0 & 0 & 100 & 0 & 0 & 0 & 100 & 0 & 0 & 0 & 100 & 0 & 0 \\
\hline & SCAIC & 0 & 99.7 & 0.3 & 0 & 0 & 99.7 & 0.3 & 0 & 0 & 99.3 & 0.7 & 0 & 0 & 99.3 & 0.7 & 0 \\
\hline & BIC & 0 & 100 & 0 & 0 & 0 & 100 & 0 & 0 & 0 & 100 & 0 & 0 & 0 & 100 & 0 & 0 \\
\hline & SBIC & 0 & 97.2 & 2.8 & 0 & 0 & 97.2 & 2.8 & 0 & 0 & 98.3 & 1.7 & 0 & 0 & 98.3 & 1.7 & 0 \\
\hline & Entropy & 0 & 54.1 & 35.7 & 10.2 & 0 & 54 & 35.8 & 10.2 & 0 & 54.1 & 35.7 & 10.2 & 0 & 54.1 & 35.7 & 10.2 \\
\hline & CLC & 0 & 0 & 0 & 100 & 0 & 0 & 0 & 100 & 0 & 0 & 0.7 & 99.3 & 0 & 0 & 0.7 & 99.3 \\
\hline & NEC & 0 & 36.5 & 19.1 & 44.4 & 0 & 36.6 & 19.2 & 44.2 & 0 & 40.5 & 23.1 & 36.4 & 0 & 40.5 & 23.1 & 36.4 \\
\hline & ICL-BIC & 0 & 100 & 0 & 0 & 0 & 100 & 0 & 0 & 0 & 100 & 0 & 0 & 0 & 100 & 0 & 0 \\
\hline \multirow[t]{9}{*}{$N=2,000$} & $\mathrm{AIC}$ & 0 & 75.3 & 20.6 & 4.1 & 0 & 75.3 & 20.6 & 4.1 & 0 & 74.6 & 22.2 & 3.2 & 0 & 74.6 & 22.2 & 3.2 \\
\hline & CAIC & 0 & 100 & 0 & 0 & 0 & 100 & 0 & 0 & 0 & 100 & 0 & 0 & 0 & 100 & 0 & 0 \\
\hline & SCAIC & 0 & 99.6 & 0.4 & 0 & 0 & 99.6 & 0.4 & 0 & 0 & 99.5 & 0.5 & 0 & 0 & 99.5 & 0.5 & 0 \\
\hline & BIC & 0 & 99.8 & 0.2 & 0 & 0 & 99.8 & 0.2 & 0 & 0 & 99.5 & 0.5 & 0 & 0 & 99.5 & 0.5 & 0 \\
\hline & SBIC & 0 & 99.5 & 0.5 & 0 & 0 & 99.5 & 0.5 & 0 & 0 & 99 & 1 & 0 & 0 & 99 & 1 & 0 \\
\hline & Entropy & 0 & 56.6 & 33.9 & 9.5 & 0 & 56.9 & 33.4 & 9.7 & 0 & 51.5 & 34.3 & 14.2 & 0 & 51.4 & 34.4 & 14.2 \\
\hline & CLC & 0 & 0 & 0.9 & 99.1 & 0 & 0 & 0.7 & 99.3 & 0 & 0 & 0.2 & 99.8 & 0 & 0 & 0.2 & 99.8 \\
\hline & NEC & 0 & 39.1 & 22.2 & 38.7 & 0 & 38.8 & 22.4 & 38.8 & 0 & 44.3 & 23.1 & 32.6 & 0 & 44.4 & 22.9 & 32.7 \\
\hline & ICL-BIC & 0 & 99.8 & 0.2 & 0 & 0 & 99.8 & 0.2 & 0 & 0 & 99.8 & 0.2 & 0 & 0 & 99.8 & 0.2 & 0 \\
\hline
\end{tabular}

TVC $=$ time-varying covariate $;$ AIC $=$ Akaike's information criterion $;$ CAIC $=$ consistent AIC $;$ SCAIC $=$ sample size adjusted CAIC BIC $=$ Bayesian information criterion; $\mathrm{SBIC}=$ sample size adjusted $\mathrm{BIC} ; \mathrm{NEC}=$ normalized entropy criterion; ICL-BIC $=$ integrated completed likelihood criterion. 
Table 5 Class enumeration accuracy of the indicators as a function of sample size, for a mixing ratio of $0.30 / 0.70$ and four time points

\begin{tabular}{|c|c|c|c|}
\hline $\begin{array}{l}\text { Homoscedastic and } \\
\text { TVC-R }{ }^{2}=2 \%\end{array}$ & $\begin{array}{l}\text { Homoscedastic and } \\
\text { TVC-R }{ }^{2}=7 \%\end{array}$ & $\begin{array}{l}\text { Heteroscedastic and } \\
\text { TVC-R }{ }^{2}=2 \%\end{array}$ & $\begin{array}{l}\text { Heteroscedastic and } \\
\text { TVC-R }{ }^{2}=7 \%\end{array}$ \\
\hline
\end{tabular}

\begin{tabular}{|c|c|c|c|c|c|c|c|c|c|c|c|c|c|c|c|c|c|}
\hline \multirow{2}{*}{$\begin{array}{l}\# \text { of Classe } \\
N=250\end{array}$} & & 1 & 2 & 3 & 4 & 1 & 2 & 3 & 4 & 1 & 2 & 3 & 4 & 1 & 2 & 3 & 4 \\
\hline & AIC & 0 & 67.2 & 27 & 5.8 & 0 & 67.4 & 26.8 & 5.8 & 0 & 69.8 & 24.6 & 5.6 & 0 & 69.8 & 24.6 & 5.6 \\
\hline & CAIC & 0 & 100 & 0 & 0 & 0 & 100 & 0 & 0 & 0 & 100 & 0 & 0 & 0 & 100 & 0 & 0 \\
\hline & SCAIC & 0 & 94.2 & 5.3 & .5 & 0 & 94.2 & 5.3 & .5 & 0 & 92.3 & 7 & 0.7 & 0 & 92.3 & 7 & 0.7 \\
\hline & BIC & 0 & 100 & 0 & 0 & 0 & 100 & 0 & 0 & 0 & 100 & 0 & 0 & 0 & 100 & 0 & 0 \\
\hline & SBIC & 0 & 77.2 & 21.7 & 1.1 & 0 & 77.4 & 21.6 & 1 & 0 & 78.9 & 18.3 & 2.8 & 0 & 78.9 & 18.3 & 2.8 \\
\hline & Entropy & 0 & 63 & 29.6 & 7.4 & 0 & 63.2 & 29.5 & 7.3 & 0 & 61.3 & 29.6 & 9.1 & 0 & 61.3 & 30.3 & 8.4 \\
\hline & CLC & 0 & 0 & 1.1 & 98.9 & 0 & 0 & 1.1 & 98.9 & 0 & 0 & 0 & 100 & 0 & 0 & 0 & 100 \\
\hline & NEC & 0 & 3.2 & 17.5 & 79.3 & 0 & 3.1 & 17.9 & 79 & 0 & 2.8 & 18.3 & 78.9 & 0 & 2.8 & 18.3 & 78.9 \\
\hline & ICL-BIC & 0 & 100 & 0 & 0 & 0 & 100 & 0 & 0 & 0 & 100 & 0 & 0 & 0 & 100 & 0 & 0 \\
\hline$N=500$ & $\mathrm{AIC}$ & 0 & 73 & 22 & 5 & 0 & 73 & 22 & 5 & 0 & 72 & 20.4 & 7.6 & 0 & 72 & 20.4 & 7.6 \\
\hline & CAIC & 0 & 100 & 0 & 0 & 0 & 100 & 0 & 0 & 0 & 100 & 0 & 0 & 0 & 100 & 0 & 0 \\
\hline & SCAIC & 0 & 99 & 1 & 0 & 0 & 99 & 1 & 0 & 0 & 98.6 & 1.4 & 0 & 0 & 98.6 & 1.4 & 0 \\
\hline & $\mathrm{BIC}$ & 0 & 100 & 0 & 0 & 0 & 100 & 0 & 0 & 0 & 100 & 0 & 0 & 0 & 100 & 0 & 0 \\
\hline & SBIC & 0 & 94.3 & 5.7 & 0 & 0 & 94.3 & 5.7 & 0 & 0 & 92.9 & 7.1 & 0 & 0 & 92.9 & 7.1 & 0 \\
\hline & Entropy & 0 & 56.1 & 36.1 & 7.8 & 0 & 56.1 & 36.1 & 7.8 & 0 & 55 & 35.5 & 9.5 & 0 & 55 & 35.5 & 9.5 \\
\hline & CLC & 0 & 0 & 0.7 & 99.3 & 0 & 0 & 0.3 & 99.7 & 0 & 0 & 0 & 100 & 0 & 0 & 0 & 100 \\
\hline & NEC & 0 & 17.9 & 21.6 & 60.5 & 0 & 17.9 & 22 & 60.1 & 0 & 18.5 & 21.8 & 59.7 & 0 & 18.5 & 21.8 & 59.7 \\
\hline & ICL-BIC & 0 & 100 & 0 & 0 & 0 & 100 & 0 & 0 & 0 & 100 & 0 & 0 & 0 & 100 & 0 & 0 \\
\hline
\end{tabular}

TVC $=$ time-varying covariate $;$ AIC $=$ Akaike's information criterion $;$ CAIC $=$ consistent AIC SCAIC $=$ sample size adjusted CAIC BIC $=$ Bayesian information criterion; SBIC = sample size adjusted BIC; NEC = normalized entropy criterion; ICL-BIC = integrated completed likelihood criterion.

from $73.13 \%$ for $N=250$ to $75.83 \%$ for $N=1,000$, with an average of $74.22 \%$ and a tendency to select the threeclass solution $20.88 \%$ of the time. In contrast, the CAIC and the BIC selected the two-class solution almost $100 \%$ of the time across conditions, and the performance of the SCAIC ranged from $94.69 \%$ for $N=250$ to $99.8 \%$ for $N$ $=2,000$, and that of the SBIC from $81.87 \%$ for $N=250$ to $99.05 \%$ for $N=2,000$. Among the entropy-based criteria, the ICL-BIC generally resulted in an accurate class enumeration across sample sizes, with an average rate of $99.80 \%$. Conversely, entropy alone was less accurate across sample sizes, with an average rate of $70.72 \%$.

Main effect of number of time points The number of time points had a large effect on the entropy $\left(\eta^{2}=.88\right)$ and AIC $\left(\eta^{2}\right.$ $=.26$ ) measures. The AIC did not perform as well as the other ICs, with rates of correct identification of the two-class solution ranging from $72.7 \%$ (for six time points) to $76.12 \%$ (for

Table 6 Class enumeration accuracy of the indicators as a function of sample size, for a mixing ratio of 0.30/0.70 and four time points

\begin{tabular}{|c|c|c|c|c|c|c|c|c|c|c|c|c|c|c|c|c|c|}
\hline \multirow[b]{2}{*}{ \# of Classes } & & \multicolumn{4}{|c|}{$\begin{array}{l}\text { Homoscedastic and } \\
\text { TVC-R }{ }^{2}=2 \%\end{array}$} & \multicolumn{4}{|c|}{$\begin{array}{l}\text { Homoscedastic and } \\
\text { TVC-R }{ }^{2}=7 \%\end{array}$} & \multicolumn{4}{|c|}{$\begin{array}{l}\text { Heteroscedastic and } \\
\text { TVC-R }{ }^{2}=2 \%\end{array}$} & \multicolumn{4}{|c|}{$\begin{array}{l}\text { Heteroscedastic and } \\
\text { TVC- } \mathrm{R}^{2}=7 \%\end{array}$} \\
\hline & & 1 & 2 & 3 & 4 & 1 & 2 & 3 & 4 & 1 & 2 & 3 & 4 & 1 & 2 & 3 & 4 \\
\hline \multirow[t]{9}{*}{$N=1,000$} & $\mathrm{AIC}$ & 0 & 76.1 & 19.9 & 4 & 0 & 76.1 & 19.9 & 4 & 0 & 75.8 & 19.9 & 4.3 & 0 & 75.8 & 19.9 & 4.3 \\
\hline & CAIC & 0 & 100 & 0 & 0 & 0 & 100 & 0 & 0 & 0 & 100 & 0 & 0 & 0 & 100 & 0 & 0 \\
\hline & SCAIC & 0 & 98.7 & 1.3 & 0 & 0 & 98.7 & 1.3 & 0 & 0 & 99 & 1 & 0 & 0 & 99 & 1 & 0 \\
\hline & BIC & 0 & 99.8 & 0.2 & 0 & 0 & 99.8 & 0.2 & 0 & 0 & 100 & 0 & 0 & 0 & 100 & 0 & 0 \\
\hline & SBIC & 0 & 97 & 3 & 0 & 0 & 97 & 3 & 0 & 0 & 96.7 & 3.3 & 0 & 0 & 96.7 & 3.3 & 0 \\
\hline & Entropy & 0 & 56.2 & 35.2 & 8.6 & 0 & 56.2 & 35.2 & 8.6 & 0 & 52.2 & 36.5 & 11.3 & 0 & 52.2 & 36.5 & 11.3 \\
\hline & CLC & 0 & 0 & 0.2 & 99.8 & 0 & 0 & 0.2 & 99.8 & 0 & 0 & 0.7 & 99.3 & 0 & 0 & 0.7 & 99.3 \\
\hline & NEC & 0 & 36.8 & 24.9 & 38.3 & 0 & 36.8 & 25.2 & 38 & 0 & 41.2 & 24.9 & 33.9 & 0 & 41.2 & 24.9 & 33.9 \\
\hline & ICL-BIC & 0 & 99.8 & 0.2 & 0 & 0 & 99.8 & 0.2 & 0 & 0 & 99.7 & 0.3 & 0 & 0 & 99.7 & 0.3 & 0 \\
\hline \multirow{9}{*}{$N=2,000$} & $\mathrm{AIC}$ & 0 & 74.6 & 22.1 & 3.3 & 0 & 74.6 & 22.1 & 3.3 & 0 & 71.3 & 25.3 & 3.4 & 0 & 71.4 & 25.2 & 3.4 \\
\hline & CAIC & 0 & 100 & 0 & 0 & 0 & 100 & 0 & 0 & 0 & 100 & 0 & 0 & 0 & 100 & 0 & 0 \\
\hline & SCAIC & 0 & 99.6 & 0.4 & 0 & 0 & 99.6 & 0.4 & 0 & 0 & 100 & 0 & 0 & 0 & 100 & 0 & 0 \\
\hline & BIC & 0 & 100 & 0 & 0 & 0 & 100 & 0 & 0 & 0 & 100 & 0 & 0 & 0 & 100 & 0 & 0 \\
\hline & SBIC & 0 & 98.3 & 1.7 & 0 & 0 & 98.3 & 1.7 & 0 & 0 & 98.3 & 1.7 & 0 & 0 & 98.3 & 1.7 & 0 \\
\hline & Entropy & 0 & 56.2 & 33.6 & 10.2 & 0 & 56.2 & 33.6 & 10.2 & 0 & 51.1 & 36 & 12.9 & 0 & 51.4 & 35.8 & 12.8 \\
\hline & CLC & 0 & 0 & 0.5 & 99.5 & 0 & 0 & 0.5 & 99.5 & 0 & 0 & 0 & 100 & 0 & 0 & 0.2 & 99.8 \\
\hline & NEC & 0 & 39.5 & 28.9 & 31.6 & 0 & 39.5 & 28.3 & 32.2 & 0 & 43.5 & 29.7 & 26.8 & 0 & 43.3 & 29.8 & 26.9 \\
\hline & ICL-BIC & 0 & 100 & 0 & 0 & 0 & 100 & 0 & 0 & 0 & 100 & 0 & 0 & 0 & 100 & 0 & 0 \\
\hline
\end{tabular}

TVC $=$ time-varying covariate $;$ AIC $=$ Akaike's information criterion $;$ CAIC $=$ consistent AIC $;$ SCAIC $=$ sample size adjusted CAIC BIC $=$ Bayesian information criterion; SBIC $=$ sample size adjusted BIC; NEC $=$ normalized entropy criterion; ICL-BIC $=$ integrated completed likelihood criterion. 
eight time points) and a tendency to erroneously select the three-class solution $20.88 \%$ of the time. The other ICs correctly selected the two-class solution almost $100 \%$ of the time. The performance of the ICL-BIC is comparable to that of the ICs, and resulted in the selection of the correct number of classes $100 \%$ of the time. In contrast, the performance of entropy remained lower, and tended to increase with the number of time points. With four time points, entropy selected the two-class solution $56.31 \%$ of the time, and the three-class solution $34.05 \%$ of the time. Comparable figures are $73.18 \%$ and $23.74 \%$ with six time points, and $78.89 \%$ and $19.29 \%$ with eight time points.

Main effects of the mixing ratio, residual structure, and $\mathbf{R}^{2}$ associated with the TVC The mixing ratio, residual structure, and the TVC- $\mathrm{R}^{2}$ conditions only had very limited effects (either small-trivial or nonsignificant) on the accuracy of the indicators. For instance, across conditions, most ICs, as well as the ICL-BIC, selected the correct number of classes more than $90 \%$ of the time, on average. The AIC and entropy did not perform as well, with average rates of correct identification of the two-class solution of $74.22 \%$ for the AIC and of $69.46 \%$ for the entropy.

Interaction effects With four time points, the accuracy increased with sample size, but the effect of sample size became negligible with more time points. Similarly, the accuracy of the BIC and of the ICL-BIC was negatively related to sample size for the unequal mixing ratio condition, but a negligible relationship was found for the equal mixing ratio condition. Finally, with four time points the AIC had a low accuracy in the unequal mixing ratio condition, whereas the effect of the mixing ratio condition became negligible with more time points.

\section{Parameter recovery}

Table 7 provides the ANOVA results for the effects of all design conditions on the levels of the biases (RPB) associated with the model parameters. More extensive information on parameter recovery is reported in Tables S10-S105 of the online supplements.

Growth factors mean As presented in Table 7, the rates of bias in the estimation of the growth factor means and of their standard errors were only influenced by the number of time points, with explained percentages of variance $\left(\eta^{2}\right)$ that varied between .97 and .99 . Across conditions, the RPB associated with the estimation of the mean of the intercept factor remained negligible $(<10 \%$; see Kaplan, 1989) for the first latent class (RPB $=-9.75 \%$ to $0.05 \%$ ), but was more pronounced for the second latent class (RPB $=0$ to $18.12 \%$ ), in which the population values for the mean of these growth factors was smaller in magnitude. Furthermore, the RPB associated with the mean of the intercept factor was higher with four time points ($5.53 \%$ to $-9.75 \%$ for the first class, and $10.27 \%$ to $13.50 \%$ for the second class) than with more time points (ranging from $-0.08 \%$ to $0.05 \%$ for the first class, and from $0 \%$ to $0.26 \%$ for the second class). However, the standard errors of the intercept means were underestimated, with more important biases associated with the four-time-point condition. The RPB of the standard errors of the intercept means varied from $-96.30 \%$ to $6.79 \%$, for the first latent class, and from $-96.56 \%$ to $1.50 \%$, for the second latent class. Due to this underestimation of the standard errors, the coverage values for the means of the intercept factors remains smaller with four time points (73.60 \% to $83.50 \%$ for the first class; $76 \%$ to $84 \%$ for the second class), but satisfactory with more time points $(93.20 \%$ to $96.40 \%$ for the first class; $93.10 \%$ to $95.90 \%$ for the second class). A similar pattern of results was noted for the means of the slope factor, although the biases associated with these parameters and their standard errors were more pronounced than those associated with the intercept means.

Growth factor variances and covariance The sample size, the number of time points, and the interaction between these two conditions had a large impact on the presence of biases in estimations of the variances of the intercept $\left(\eta^{2}=.58\right.$ for sample size, .18 for time points, and .17 for their interaction) and slope $\left(\eta^{2}=.41\right.$ for sample size, .22 for time points, and .33 for their interaction) parameters, as well as of their covariance $\left(\eta^{2}=.39\right.$ for sample size, .25 for time points, and .29 for their interaction). These same factors generally had effects of a similar magnitude on the estimation of the standard errors of these parameters $\left(\eta^{2}\right.$ varying between .03 and .63). Other design factors had only a negligible $\left(\eta^{2} \leq .06\right)$ or a nonsignificant impact on these biases. Importantly, the results show that the largest bias was found for four time points and small sample size. However, increases in both the sample size and the number of time points tended to compensate one another to reduce the biases associated with the estimations of these model parameters.

To summarize these effects, the models performed relatively well in terms of parameter recovery associated with the variance of the intercept factor $(\mathrm{RPB}=-$ $2.82 \%$ to $0.09 \%$; coverage $=93.20 \%$ to $96 \%$ ) and of its standard error ( $\mathrm{RPB}=-3.35 \%$ to $4.50 \%$ ) across conditions. Although the effects of sample size, number of time points, and their interaction on the biases associated with the variance of the slope factor were important, the RPB and rates of coverage themselves remained satisfactory across conditions. In contrast, a slight underestimation, which was higher with fewer time points and smaller sample sizes, was observed for the variance of the slope factor (RPB $=-19.56 \%$ to $0.34 \%$ across conditions). Although these results display similar tendencies across 
Table $7 \mathrm{Eta}^{2}$ of the ANOVA associated with biases in parameter recovery as function of design conditions

\begin{tabular}{|c|c|c|c|c|c|c|c|c|}
\hline Factors & $\begin{array}{l}\text { Class } 1 \\
\mu_{\alpha} \text { RPB }\end{array}$ & S.E. & $\begin{array}{l}\text { Class } 2 \\
\mu_{\alpha} \mathrm{RPB}\end{array}$ & S.E. & $\begin{array}{l}\text { Class } 1 \\
\mu_{\beta} \mathrm{RPB}\end{array}$ & S.E. & $\begin{array}{l}\text { Class } 2 \\
\mu_{\beta} \mathrm{RPB}\end{array}$ & S.E. \\
\hline Sample size (A) & 0 & 0 & 0 & 0 & 0 & 0 & 0 & 0 \\
\hline Time points (B) & 0.98 & 0.99 & 0.98 & 0.99 & 0.97 & 0.99 & 0.98 & 0.99 \\
\hline Mixing ratio (C) & 0 & 0 & 0 & 0 & 0 & 0 & 0 & 0 \\
\hline Residuals (D) & 0 & 0 & 0 & 0 & 0 & 0 & 0 & 0 \\
\hline TVC-R $^{2}(\mathrm{E})$ & 0 & 0 & 0 & 0 & 0 & 0 & 0 & 0 \\
\hline $\mathrm{A} * \mathrm{~B}$ & 0.01 & 0 & 0.01 & 0.01 & 0.01 & 0.01 & 0 & 0.01 \\
\hline $\mathrm{A}^{*} \mathrm{C}$ & 0 & 0 & 0 & 0 & 0 & 0 & 0 & 0 \\
\hline$A * D$ & 0 & 0 & 0 & 0 & 0 & 0 & 0 & 0 \\
\hline $\mathrm{A}^{*} \mathrm{E}$ & 0 & 0 & 0 & 0 & 0 & 0 & 0 & 0 \\
\hline $\mathrm{B} * \mathrm{C}$ & 0 & 0 & 0 & 0 & 0 & 0 & 0 & 0 \\
\hline$B * D$ & 0 & 0 & 0 & 0 & 0 & 0 & 0 & 0 \\
\hline$B * E$ & 0 & 0 & 0 & 0 & 0 & 0 & 0 & 0 \\
\hline$C * D$ & 0 & 0 & 0 & 0 & 0 & 0 & 0 & 0 \\
\hline$C^{*} \mathrm{E}$ & 0 & 0 & 0 & 0 & 0 & 0 & 0 & 0 \\
\hline \multirow[t]{2}{*}{ D*E } & 0 & 0 & 0 & 0 & 0 & 0 & 0 & 0 \\
\hline & $\psi_{\alpha y \alpha y} \mathrm{RPB}$ & S.E. & $\psi_{\beta y \beta y} \mathrm{RPB}$ & S.E. & $\psi_{\alpha y \beta y} \mathrm{RPB}$ & S.E. & $p^{1} \mathrm{RPB}$ & S.E. \\
\hline Sample size (A) & 0.58 & 0.03 & 0.41 & 0.34 & 0.39 & 0.09 & 0 & 0 \\
\hline Time points (B) & 0.18 & 0.22 & 0.22 & 0.11 & 0.25 & 0.61 & 0.40 & 0.40 \\
\hline Mixing ratio $(\mathrm{C})$ & 0 & 0.01 & 0 & 0 & 0 & 0 & 0.20 & 0.18 \\
\hline Residuals (D) & 0.02 & 0.01 & 0.01 & 0.02 & 0.02 & 0 & 0 & 0 \\
\hline TVC-R $^{2}(\mathrm{E})$ & 0 & 0 & 0 & 0 & 0 & 0 & 0 & 0 \\
\hline $\mathrm{A} * \mathrm{~B}$ & 0.17 & 0.63 & 0.33 & 0.48 & 0.29 & 0.27 & 0 & 0 \\
\hline $\mathrm{A}^{*} \mathrm{C}$ & 0 & 0.01 & 0 & 0 & 0 & 0 & 0 & 0 \\
\hline$A^{*} \mathrm{D}$ & 0.02 & 0.01 & 0.01 & 0.01 & 0.01 & 0 & 0 & 0 \\
\hline$A^{*} \mathrm{E}$ & 0 & 0 & 0 & 0 & 0 & 0 & 0 & 0 \\
\hline $\mathrm{B} * \mathrm{C}$ & 0 & 0.02 & 0 & 0.01 & 0 & 0 & 0.40 & 0.42 \\
\hline$B * D$ & 0.03 & 0.01 & 0.01 & 0.01 & 0.03 & 0 & 0 & 0 \\
\hline$B * E$ & 0 & 0 & 0 & 0 & 0 & 0 & 0 & 0 \\
\hline$C * D$ & 0 & 0 & 0 & 0 & 0 & 0 & 0 & 0 \\
\hline $\mathrm{C} * \mathrm{E}$ & 0 & 0 & 0 & 0 & 0 & 0 & 0 & 0 \\
\hline \multirow[t]{2}{*}{$\mathrm{D} * \mathrm{E}$} & 0 & 0 & 0 & 0 & 0 & 0 & 0 & 0 \\
\hline & $\gamma_{1}$ RPB & S.E. & $\gamma_{2}$ RPB & S.E. & $\gamma_{3} \mathrm{RPB}$ & S.E. & $\gamma_{4} \mathrm{RPB}$ & S.E. \\
\hline Sample size (A) & 0.06 & 0.35 & 0.03 & 0.20 & 0.12 & 0.48 & 0.42 & 0.06 \\
\hline Time points (B) & 0.57 & 0.23 & 0.03 & 0.08 & 0.12 & 0 & 0.20 & 0.66 \\
\hline Mixing ratio (C) & 0 & 0 & 0.75 & 0 & 0.05 & 0 & 0.02 & 0 \\
\hline Residuals (D) & 0 & 0 & 0 & 0 & 0 & 0 & 0 & 0 \\
\hline TVC-R $^{2}(\mathrm{E})$ & 0.01 & 0 & 0 & 0 & 0.01 & 0 & 0.06 & 0 \\
\hline $\mathrm{A} * \mathrm{~B}$ & 0.27 & 0.41 & 0.13 & 0.72 & 0.73 & 0.52 & 0.21 & 0.28 \\
\hline $\mathrm{A}^{*} \mathrm{C}$ & 0 & 0 & 0 & 0 & 0 & 0 & 0 & 0 \\
\hline$A * D$ & 0 & 0 & 0 & 0 & 0 & 0 & 0 & 0 \\
\hline$A * E$ & 0 & 0 & 0 & 0 & 0.01 & 0 & 0.04 & 0 \\
\hline $\mathrm{B}^{*} \mathrm{C}$ & 0 & 0 & 0 & 0 & 0 & 0 & 0.01 & 0 \\
\hline$B * D$ & 0 & 0 & 0 & 0 & 0 & 0 & 0 & 0 \\
\hline B*E & 0.06 & 0 & 0.06 & 0 & 0 & 0 & 0.02 & 0 \\
\hline $\mathrm{C} * \mathrm{D}$ & 0 & 0 & 0 & 0 & 0 & 0 & 0 & 0 \\
\hline$C * E$ & 0 & 0 & 0 & 0 & 0 & 0 & 0 & 0 \\
\hline \multirow[t]{2}{*}{$\mathrm{D}^{*} \mathrm{E}$} & 0 & 0 & 0 & 0 & 0 & 0 & 0 & 0 \\
\hline & $\gamma_{5} \mathrm{RPB}$ & S.E. & $\gamma_{6} \mathrm{RPB}$ & S.E. & $\gamma_{7} \mathrm{RPB}$ & S.E. & $\gamma_{8} \mathrm{RPB}$ & S.E. \\
\hline Sample size (A) & 0.24 & 0.17 & 0.59 & 0.16 & 0.89 & 0.99 & 0.67 & 0.99 \\
\hline Time points (B) & 0.16 & 0.34 & 0.17 & 0.54 & 0 & 0 & 0 & 0 \\
\hline Mixing ratio (C) & 0 & 0 & 0 & 0 & 0 & 0 & 0 & 0 \\
\hline Residuals (D) & 0 & 0 & 0 & 0 & 0 & 0 & 0.01 & 0 \\
\hline TVC-R ${ }^{2}(E)$ & 0.06 & 0 & 0.10 & 0 & 0.02 & 0.01 & 0.25 & 0 \\
\hline $\mathrm{A} * \mathrm{~B}$ & 0.46 & 0.48 & 0.06 & 0.30 & 0 & 0 & 0 & 0 \\
\hline $\mathrm{A}^{*} \mathrm{C}$ & 0 & 0 & 0 & 0 & 0 & 0 & 0 & 0 \\
\hline$A * D$ & 0 & 0 & 0 & 0 & 0.09 & 0 & 0 & 0 \\
\hline$A^{*} \mathrm{E}$ & 0.02 & 0 & 0.06 & 0 & 0 & 0 & 0.07 & 0 \\
\hline $\mathrm{B} * \mathrm{C}$ & 0 & 0 & 0 & 0 & 0 & 0 & 0 & 0 \\
\hline $\mathrm{B} * \mathrm{D}$ & 0 & 0 & 0 & 0 & 0 & 0 & 0 & 0 \\
\hline $\mathrm{B} * \mathrm{E}$ & 0.02 & 0 & 0.02 & 0 & 0 & 0 & 0 & 0 \\
\hline$C * D$ & 0 & 0 & 0 & 0 & 0 & 0 & 0 & 0 \\
\hline $\mathrm{C} * \mathrm{E}$ & 0 & 0 & 0 & 0 & 0 & 0 & 0 & 0 \\
\hline \multirow[t]{2}{*}{$\mathrm{D} * \mathrm{E}$} & 0 & 0 & 0 & 0 & 0 & 0 & 0 & 0 \\
\hline & $\theta_{1} \mathrm{RPB}$ & S.E. & $\theta_{2} \mathrm{RPB}$ & S.E. & $\theta_{3} \mathrm{RPB}$ & S.E. & $\theta_{4} \mathrm{RPB}$ & S.E. \\
\hline Sample size (A) & 0.05 & 0.20 & 0.47 & 0.53 & 0.28 & 0.16 & 0.23 & 0.13 \\
\hline
\end{tabular}


Table 7 (continued)

\begin{tabular}{|c|c|c|c|c|c|c|c|c|}
\hline $\begin{array}{l}\text { Time points } \\
\text { (B) }\end{array}$ & 0.59 & $\begin{array}{l}0.1- \\
1\end{array}$ & 0.43 & $\begin{array}{l}0.0- \\
9\end{array}$ & 0.25 & $\begin{array}{l}0.5- \\
6\end{array}$ & 0.74 & $\begin{array}{l}0.1- \\
4\end{array}$ \\
\hline $\begin{array}{l}\text { Mixing ratio } \\
\text { (C) }\end{array}$ & 0 & $\begin{array}{l}0.0- \\
1\end{array}$ & 0 & 0 & 0 & $\begin{array}{l}0.0- \\
1\end{array}$ & 0 & $\begin{array}{l}0.0- \\
2\end{array}$ \\
\hline Residuals (D) & 0 & $\begin{array}{l}0.0- \\
3\end{array}$ & 0 & 0 & 0 & 0 & 0 & 0 \\
\hline TVC-R ${ }^{2}(\mathrm{E})$ & 0 & 0 & 0 & 0 & 0 & 0 & 0 & 0 \\
\hline $\mathrm{A} * \mathrm{~B}$ & 0.35 & $\begin{array}{l}0.6- \\
0\end{array}$ & 0.10 & $\begin{array}{l}0.3- \\
7\end{array}$ & 0.46 & $\begin{array}{l}0.2- \\
6\end{array}$ & 0.01 & $\begin{array}{l}0.6- \\
7\end{array}$ \\
\hline $\mathrm{A}^{*} \mathrm{C}$ & 0 & 0 & 0 & 0 & 0 & 0 & 0 & 0 \\
\hline$A * D$ & 0 & $\begin{array}{l}0.0- \\
1\end{array}$ & 0 & 0 & 0 & 0 & 0 & 0 \\
\hline$A^{*} \mathrm{E}$ & 0 & 0 & 0 & 0 & 0 & 0 & 0 & 0 \\
\hline $\mathrm{B} * \mathrm{C}$ & 0 & 0 & 0 & 0 & 0 & $\begin{array}{l}0.0- \\
1\end{array}$ & 0 & $\begin{array}{l}0.0- \\
2\end{array}$ \\
\hline $\mathrm{B} * \mathrm{D}$ & 0 & $\begin{array}{l}0.0- \\
1\end{array}$ & 0 & 0 & 0 & 0 & 0 & 0 \\
\hline $\mathrm{B} * \mathrm{E}$ & 0 & 0 & 0 & 0 & 0 & 0 & 0 & 0 \\
\hline$C * D$ & 0 & 0 & 0 & 0 & 0 & 0 & 0 & 0 \\
\hline $\mathrm{C} * \mathrm{E}$ & 0 & 0 & 0 & 0 & 0 & 0 & 0 & 0 \\
\hline$D^{*} \mathrm{E}$ & $\begin{array}{l}0 \\
\theta_{5} \mathrm{RPB}\end{array}$ & $\begin{array}{l}0 \\
\text { S.- } \\
\text { E. }\end{array}$ & $\begin{array}{l}0 \\
\theta_{6} \mathrm{RPB}\end{array}$ & $\begin{array}{l}0 \\
\text { S.- } \\
\text { E. }\end{array}$ & $\begin{array}{l}0 \\
\theta_{7} \mathrm{RPB}\end{array}$ & $\begin{array}{l}0 \\
\text { S.- } \\
\text { E. }\end{array}$ & $\begin{array}{l}0 \\
\theta_{8} \mathrm{RPB}\end{array}$ & $\begin{array}{l}0 \\
\text { S.- } \\
\text { E. }\end{array}$ \\
\hline $\begin{array}{l}\text { Sample size } \\
\text { (A) }\end{array}$ & 0.13 & $\begin{array}{l}0.1- \\
4\end{array}$ & 0.44 & $\begin{array}{l}0.1- \\
4\end{array}$ & 0.01 & $\begin{array}{l}0.0- \\
8\end{array}$ & 0.25 & $\begin{array}{l}0.0- \\
2\end{array}$ \\
\hline $\begin{array}{l}\text { Time points } \\
\text { (B) }\end{array}$ & 0.13 & $\begin{array}{l}0.1- \\
0\end{array}$ & 0.31 & $\begin{array}{l}0.0- \\
2\end{array}$ & 0.95 & $\begin{array}{l}0.7- \\
4\end{array}$ & 0.17 & $\begin{array}{l}0.9- \\
4\end{array}$ \\
\hline $\begin{array}{l}\text { Mixing ratio } \\
\text { (C) }\end{array}$ & 0 & 0 & 0.01 & 0 & 0 & 0 & 0 & 0 \\
\hline Residuals (D) & 0 & $\begin{array}{l}0.0- \\
1\end{array}$ & 0 & 0 & 0 & 0 & 0.01 & 0 \\
\hline TVC-R $^{2}(\mathrm{E})$ & 0 & 0 & 0 & 0 & 0 & 0 & 0 & 0 \\
\hline $\mathrm{A} * \mathrm{~B}$ & 0.71 & $\begin{array}{l}0.7- \\
4\end{array}$ & 0.24 & $\begin{array}{l}0.8- \\
2\end{array}$ & 0.02 & $\begin{array}{l}0.1- \\
6\end{array}$ & 0.50 & $\begin{array}{l}0.0- \\
3\end{array}$ \\
\hline $\mathrm{A}^{*} \mathrm{C}$ & 0 & 0 & 0 & 0 & 0 & 0 & 0 & 0 \\
\hline$A * D$ & 0 & 0 & 0 & 0 & 0 & 0 & 0.01 & 0 \\
\hline$A * E$ & 0 & 0 & 0 & 0 & 0 & 0 & 0 & 0 \\
\hline$B * C$ & 0.01 & 0 & 0.01 & 0 & 0 & 0 & 0 & 0 \\
\hline$B * D$ & 0 & $\begin{array}{l}0.0- \\
1\end{array}$ & 0 & 0 & 0 & $\begin{array}{l}0.0- \\
1\end{array}$ & 0 & 0 \\
\hline$B * E$ & 0 & 0 & 0 & 0 & 0 & 0 & 0 & 0 \\
\hline$C * D$ & 0 & 0 & 0 & 0 & 0 & 0 & 0.02 & 0 \\
\hline$C^{*} \mathrm{E}$ & 0 & 0 & 0 & 0 & 0 & 0 & 0 & 0 \\
\hline $\mathrm{D}^{*} \mathrm{E}$ & 0 & 0 & 0 & 0 & 0 & 0 & 0 & 0 \\
\hline
\end{tabular}

$\mathrm{RPB}=$ relative percentage bias, S.E.: RPB of the standard error of the parameter estimate, $\mathrm{TVC}=$ time-varying covariate, $\mu_{\alpha}=$ intercept mean, $\mu_{\beta}=$ linear slope mean, $\psi_{\alpha y \alpha y}=$ intercept variance, $\psi_{\beta y \beta y}=$ linear slope variance, $\psi_{\alpha y \beta y}=$ intercept/slope covariance, $p^{1}=$ class proportions, $\gamma_{i}=$ slope of the time variant covariate at time point $i, \theta \mathrm{i}=$ residuals variance at time point $\mathrm{i}$.

conditions, the biases observed in the estimation of the standard errors of the slope variance (RPB $=-4.96 \%$ to $3.80 \%$ across conditions), as well as the rates of coverage (93.00\% to $96.00 \%$ across conditions), remained satisfactory. Finally, an important tendency toward overestimation (marked with only four time points and sample sizes lower than 2,000) was noted in the estimation of the intercept-slope covariance $(\mathrm{RPB}=-3.67 \%$ to $557.67 \%$ across conditions). Despite these biases, the standard errors ( $\mathrm{RPB}=-5.61 \%$ to $4.35 \%$ ), as well as the rates of coverage $(93.20 \%$ to $96.20 \%$ across conditions), remained satisfactory.
Class proportion The number of time points, the mixing ratio conditions, and the interaction between these two conditions had a relatively large impact on the presence of biases in the estimation of the class proportion parameter $\left(\eta^{2}=.40\right.$ for time points, .20 for mixing ratio, and .40 for their interaction) and of its standard error (with $\eta^{2}=.40$ for time points, .18 for mixing ratio, and .42 for their interaction). None of the other design factors or interactions had a significant impact on the presence of biases in the estimation of this parameter. The unequal mixing ratio condition combined with the availability of only four time points led to the most severe underestimation biases for the class proportion parameter $(\mathrm{RPB}=-42.00 \%$ to - 
$33.00 \%$; the RPB could not be computed in the equal mixing ratio condition, given the division by 0 ) and its standard error ( $\mathrm{RPB}=91.90 \%$ to $-75.82 \%$; vs. $-1.79 \%$ to $2.90 \%$ for an equal mixing ratio), as well as poor coverage values (75.00\% to $80.00 \%$; vs. $94.00 \%$ to $96.00 \%$ for an equal mixing ratio). In comparison, the biases were far less pronounced across mixing ratio conditions when more than four time points were available (class proportion RPB, $-0.16 \%$ to $0.22 \%$; standard error RPB, $-2.71 \%$ to $3.15 \%$; coverage, $93.00 \%$ to $96.00 \%$ ).

Regression slope of the repeated measure on the TVC The models performed relatively well in terms of the parameter recovery associated with the regression coefficients of the repeated measures on the TVC. The factors that had the most marked effects were the sample size $\left(\eta^{2}=.03\right.$ to .89 for the regression coefficients with a slight tendency to increase over time, and .06 to .99 for their standard errors), number of time points $\left(\eta^{2}=0\right.$ to .57 for the regression coefficients, and 0 to .66 for their standard errors), and their interaction $\left(\eta^{2}=.06\right.$ to .73 for the regression coefficients, and 0 to .72 for their standard errors). The mixing ratio $\left(\eta^{2}=.75\right)$ also had a significant impact on the biases associated with estimation of the regression coefficient associated with the second time point, but not with the other time points or standard errors. The $R^{2}$ associated with the TVC also had some, limited impact on the biases associated with the estimation of the regression coefficients $\left(\eta^{2}=0\right.$ to .25$)$, but not of their standard errors. Although a few other main effects or interactions turned out to be significant, their impact remained either negligible $\left(\eta^{2} \leq .06\right)$ or moderately small but inconsistent across time points $\left(.06 \leq \eta^{2} \leq .09\right)$, and generally trivial when examined closely. To summarize these effects, the estimates of all regression coefficients of the repeated measures on the TVC (RPB: $-2.16 \%$ to $1.64 \%$ ), and their corresponding standard errors (RPB: $-5.84 \%$ to $5.92 \%$ ), present negligible biases and high coverage values $(92.70 \%$ to $96.50 \%$ ) across conditions. Although the effects of sample size, time points, and their interaction, as well as of the TVC$\mathrm{R}^{2}$ and mixing ratio conditions, on the biases associated with the regressions of the repeated measures on the TVC were noteworthy, the RPBs and rates of coverage themselves remained satisfactory across conditions. Once again, parameter and standard error estimates tended to be more biased with four time points and small sample sizes.

Residual variances The models performed relatively well in terms of parameter recovery associated with the residuals of the repeated measures. The factors that had the most marked effects on these residuals and their standard errors were the sample size $\left(\eta^{2}=.01\right.$ to .47 for the residuals, and .02 to .53 for their standard errors), number of time points $\left(\eta^{2}=.13\right.$ to .95 for the residuals, and .02 to .94 for their standard errors), and their interaction $\left(\eta^{2}=\right.$ .01 to .71 for the residuals, and .03 to .82 for their standard errors). Although a few other effects or interactions turned out to be significant, their impacts remained trivial $\left(\eta^{2} \leq .06\right)$ and inconsistent across time points. To summarize these effects, the estimates of all residuals parameters (RPB: $-0.97 \%$ to $0.39 \%$ ), and their corresponding standard errors (RPB: $-4.65 \%$ to $5.24 \%$ ), presented negligible biases and high coverage values $(92.10 \%$ to $96.70 \%$ ) across conditions. Although the effects of sample size, number of time points, and their interaction on the biases associated with the residuals were important, the RPB and rates of coverage themselves remained satisfactory across conditions. Once again, parameter and standard error estimates tended to be more biased with four time points and small sample size.

\section{Discussion}

In this study, we evaluated parameter recovery and class enumeration accuracy for GMMs including TVCs. To do so, we considered the impacts of five design factors: sample size, number of time points, mixing ratio, residual structure, and proportion of the total variance of the repeated measures explained by the TVC. Although the impact of the sample size and mixing ratio conditions on the class enumeration accuracy of GMMs have been documented in previous simulation studies (Li \& Hser 2011; Nylund et al., 2007; Peugh \& Fan, 2012; Tofighi \& Enders, 2007), the impact of the other design factors has not been as well researched. Our results showed that the design factors that exerted the most critical effects on both the class enumeration accuracy and the rates of parameter recovery were the sample size and the number of available time points. Surprisingly, this last condition has received very limited attention in previous studies. Conversely, and contrary to our expectations, the mixing ratio, error structure, and TVC$\mathrm{R}^{2}$ conditions only had a minimal, or negligible, impact on both the class enumeration accuracy and parameter recovery of GMMs with TVCs.

More precisely, the estimation of the growth factor means and of their standard errors was only influenced by the number of time points. The mean of the intercept factor showed negligible bias for the first class but was overestimated for the second latent class, with a severe underestimation of the corresponding standard errors. The resulting coverage values were smaller. Bias was more severe with few time points (i.e., four). A similar pattern of results was seen for the mean of the slope factor. However, the biases of the slope means for the two latent classes and of their corresponding standard errors tended to be higher than those of the intercept means. Similarly, the number of time points, the mixing ratio condition, and the interaction between these two design factors had a relatively large impact on the estimations of the class proportion parameter 
and of its standard error. Severe underestimation occurred for the class proportion parameter and its standard errors in the unequal mixing ratio condition and four time points. In contrast, negligible bias and high coverage values were noted for the following parameters and their standard errors: the growth factors' variances and covariance, the regression slope of the repeated measure on the TVC, and the residual variances. The sample size, the number of time points, and the interaction between these two conditions had an impact on the estimation of these parameters and of their corresponding standard errors.

In terms of class enumeration accuracy, our results were particularly encouraging, showing that most of the indicators had a high level of accuracy across all design conditions. In particular, and supporting the conclusions from most previous studies (Li \& Hser, 2011; Liu \& Hancock, 2014; Nylund et al., 2007; Tofighi \& Enders, 2007; Tolvanen, 2008), the BIC and CAIC proved to be particularly reliable indicators of the number of latent classes across all design conditions. Similarly, the SCAIC and ICL-BIC - two indicators that have received less attention in previous studies (e.g., Peugh \& Fan, 2012, 2013, 2015) — also proved to be very reliable. The SBIC also proved to be efficient at identifying the true number of latent classes, and its efficacy increased with sample size, again supporting the results from previous GMM simulation studies (Li \& Hser, 2011; Peugh \& Fan, 2015; Tolvanen, 2008). Altogether, these indices all selected the correct number of latent classes in the data more than $90 \%$ of the time across design conditions.

Other indicators did not perform as well. For instance, confirming the conclusions from numerous studies ( $\mathrm{Li} \&$ Hser, 2011; Nylund et al., 2007; Peugh \& Fan, 2013, 2015; Tofighi \& Enders, 2007), the AIC proved to be the least reliable of the ICs (with a correct class enumeration rate of $74.22 \%$ across conditions), and the one most sensitive to design conditions. Similarly, the entropy (with a correct class enumeration rate of $69.5 \%$ across conditions) proved to be unsatisfactory and highly sensitive to the design conditions. This result supports previous suggestions that the entropy should only be used as a descriptor of the classification accuracy of a mixture solution, rather than to identify the optimal number of latent classes in the data (Lubke \& Muthén, 2007; Peugh \& Fan, 2012, 2013). Finally, both the NEC and CLC proved inappropriate indicators of the true number of latent classes, with rates of correct identification of the two-class solution that were respectively equal to $16.21 \%$ and $0 \%$. However, previous simulation studies in which homogeneous population models (including a single latent class) were considered suggested that both the CLC and ICL-BIC (the entropy and NEC are undefined for a one-class solutions) were particularly efficient at identifying the presence of a single latent class of participants in the data (Peugh \& Fan, 2012, 2013). Future studies considering homogeneous population models would do well to systematically investigate the performance of the CLC, irrespective of its disappointing performance in the present study.

Overall, our findings show that the parameter and standard error biases, as well as convergence difficulties, are sufficiently severe to call into question the estimation of GMMs with TVCs in applied research settings when only four measurement points are available, unless the sample size is high enough to compensate for this important limitation $(N \geq 1,000$, but ideally at least 2,000). These results also have important implications for the design of future GMM simulation studies. Indeed, many previous GMM simulation studies relied on four time points without systematically varying this design condition (Nylund et al., 2007; Peugh \& Fan, 2012, 2015), or used four time points as a normative condition in a partial factorial design (Tofighi \& Enders, 2007). This decision has been mostly justified by the observation that the number of measurement occasions had only a negligible impact on the class enumeration accuracy of GMMs (Tofighi \& Enders, 2007), an observation that is mostly supported in the present study. More precisely, our results showed that although the number of time points, the sample size, and their interaction did have significant effects on the ability to retain the correct number of latent classes in the data, the class enumeration accuracy of GMMs still remained satisfactory across design conditions. However, these design factors also had a large and nonnegligible impact on the accuracy of the parameter estimates, showing that future GMM simulation studies should systematically include the number of observations as a critical design condition.

Given our objective of exploring this wider range of design conditions, while doing so in the context of a first investigation of GMMs involving TVCs, we elected to keep the range of conditions considered relatively simple. As such, we focused on multivariate normal population models including two classes, characterized by linear growth, including TVCs specified as having an impact that was constant over measurement points and across classes, and in which the residuals were also specified to be invariant across classes. Furthermore, apart from our estimation of models including different numbers of classes, in order to evaluate the class enumeration accuracy of the models, all of the models were otherwise correctly specified. The impact of varying these additional design conditions, which have also been shown to influence the performance of GMMs (Bauer \& Curran, 2003; Li \& Hser, 2011; Peugh \& Fan, 2012; Tofighi \& Enders, 2007), should be explored in the context of future studies in which the impact of including or excluding active or inactive TVCs, as well as of misspecifying their effects on the repeated measures, could also be investigated. The homoscedastic and heteroscedastic residual structures considered here were specified to be invariant across classes. However, future studies should investigate conditions of noninvariant error 
structure, as well other residual structures associated with longitudinal data, such as autoregressive structures.

In sum, our results suggest that GMMs with TVCs perform relatively well when more than four repeated measures are available, when the sample size is large, or preferably both. In practical terms, these conclusions suggest that researchers interested in the application of these models would do well to invest in increasing their sample sizes and numbers of repeated measures, which is not always feasible. This conclusion does not comes as a huge surprise, given the fact that most types of mixture models, such as GMMs with TVCs, rely on the expectation-maximization algorithm, which possesses a number of well-documented drawbacks, including slow convergence, suboptimal local optima, and poor performance with small sample sizes (Redner \& Walker, 1984). Keeping in mind that it is not always possible to increase the sample size or the number of measurement occasions, some authors (Liu \& Hancock, 2014; Pastor \& Gagné, 2013) noted that in practice, an important challenge is to achieve a balance between parsimony and complexity. GMMs with TVCs are very complex models and, as such, might benefit from the inclusion of some degree of parsimony, especially in situations in which the sample size and/or number of repeated measures are suboptimal. Referring to the estimation of GMMs more generally, Diallo, Morin, and Lu (2016) suggested that researchers should always start the class enumeration process using a theoretically optimal model, and then slowly incorporate parsimony into the model (i.e., by constraining some parameters to equality across classes or time points) when they encounter convergence problems or inadmissible solutions. Similarly, in the context of GMMs with TICs, Diallo, Morin, and $\mathrm{Lu}$ (in press) noted that it might sometimes be preferable to conduct the class enumeration process while excluding covariates, and to include these only once the optimal number of classes has been selected. Arguably, none of these recommendations has been developed specifically for GMMs with TVCs. Therefore, future research could consider the applicability of these recommendations in GMMs with TVCs, particularly when fewer than four repeated measures and 2,000 participants are available. Alternatively, Bayesian estimation methods provide another exciting alternative to the estimation of GMMs with TVCs with smaller samples or a reduced number of observations. Moreover, many of the parameter estimation problems that arise in the mixture context using maximum likelihood (or Bayes with flat prior distributions) can be fixed with the use of weakly informative prior distributions (e.g., Depaoli, 2013). This attractive approach should therefore be considered in future studies.

\section{References}

Akaike, H. (1987). Factor analysis and AIC. Psychometrika, 52, $317-$ 332.

Bauer, D. J., \& Curran, P. J. (2003). Distributional assumptions of growth mixture models: Implications for overextraction of latent trajectory classes. Psychological Methods, 8, 338-363. doi:10.1037/1082-989 X.8.3.338

Biernacki, C., Celeux, G., \& Govaert, G. (2000). Assessing a mixture model for clustering with the integrated completed likelihood. IEEE Transactions on Pattern Analysis and Machine Intelligence, 22, 719-725.

Biernacki, C., \& Govaert, G. (1997). Using the classification likelihood to choose the number of clusters. Computing Science and Statistics, 29, 451-457.

Bollen, K. A., \& Curran, P. J. (2006). Latent curve models: A structural equation perspective. New York, NY: Wiley.

Bozdogan, H. (1987). Model selection and Akaike's information criterion (AIC): The general theory and its analytical extensions. Psychometrika, 52, 345-370.

Celeux, G., \& Soromenho, G. (1996). An entropy criterion for assessing the number of clusters in a mixture model. Journal of Classification, 13, 195-212.

Chen, F., Bollen, K. A., Paxton, P., Curran, P. J., \& Kirby, J. B. (2001). Improper solutions in structural equation models: Causes, consequences, and strategies. Sociological Methods and Research, 29, 468-508

Cheong, J. (2011). Accuracy of estimates and statistical power for testing mediation in latent growth curve modeling. Structural Equation Modeling, 18, 195-211. doi:10.1080/10705511.2011.557334

Cohen, J. (1988). Statistical power analysis for behavioral sciences (2nd ed.). Hillsdale, NJ: Erlbaum.

Depaoli, S. (2013). Mixture class recovery in GMM under varying degrees of class separation: Frequentist versus Bayesian estimation. Psychological Methods, 18, 186-219. doi:10.1037/a0031609

Diallo, T. M. O., \& Morin, A. J. S. (2015). Power of latent growth curve models to detect piecewise linear trajectories. Structural Equation Modeling, 22, 449-460. doi:10.1080/10705511.2014.935678

Diallo, T. M. O., Morin, A. J. S., \& Lu, H. (2016). Impact of misspecifications of the latent variance-covariance and residual matrices on the class enumeration accuracy of growth mixture models. Structural Equation Modeling, 23, 507-531.

Diallo, T. M. O., Morin, A. J. S., \& Lu, H. (in press).The impact of total and partial inclusion or exclusion of active and inactive time invariant covariates in growth mixture models. Psychological Methods. doi: $10.1037 /$ met0000084

Diallo, T. M. O., Morin, A. J. S., \& Parker, P. D. (2014). Statistical power of latent growth curve models to detect quadratic growth. Behavior Research Methods, 46, 357-371. doi:10.3758/s13428-013-0395-1

Enders, C. K., \& Tofighi, D. (2008). The impact of miss-specifying classspecific residual variances in growth mixture models. Structural Equation Modeling, 15, 75-95.

Fan, X., \& Fan, X. (2005). Power of latent growth modeling for detecting linear growth: Number of measurements and comparison with other analytical approaches. Journal of Experimental Education, 73, 121139.

Fanti, K. A., \& Henrich, C. C. (2010). Trajectories of pure and co-occurring internalizing and externalizing problems from age 2 to age 12: Findings from the National Institute of Child Health and Human Development Study of Early Child Care. Developmental Psychology, 46, 1159-1175. doi:10.1037/a0020659

Grimm, K. J., Ram, N., \& Estabrook, R. (2010). Nonlinear structured growth mixture models in Mplus and OpenMx. Multivariate Behavioral Research, 45, 887-909. 
Hoogland, J. J., \& Boomsma, A. (1998). Robustness studies in covariance structure modeling: An overview and a meta-analysis. Sociological Methods and Research, 26, 329-367.

Kaplan, D. (1989). A study of the sampling variability and $z$-values of parameter estimates from misspecified structural equation models. Multivariate Behavioral Research, 24, 41-57.

Kim, S.-Y.(2012). Sample size requirements in single- and multiphase growth mixture models: A Monte Carlo simulation study. Structural Equation Modeling, 19, 457-476. doi:10.1080/10705511.2012.687672

Kim, S.-Y. (2014). Determining the number of latent classes in single-and multi-phase growth mixture models. Structural Equation Modeling, 21, 263-279.

Li, L., \& Hser, Y.-I. (2011). On inclusion of covariates for class enumeration of growth mixture models. Multivariate Behavioral Research, 46, 266-302.

Liang, J., Xu, X., Bennett, J. M., Ye, W., \& Quiñones, A. R. (2009). Ethnicity and changing functional health in middle and late life: A person-centered approach. Journals of Gerontology, 65B, 470-481.

Liu, M., \& Hancock, G. R. (2014). Unrestricted mixture models for class identification in growth mixture modeling. Educational and Psychological Measurement, 74, 557-584.

Lubke, G. H., \& Muthén, B. O. (2007). Performance of factor mixture models as a function of model size, covariate effects, and classspecific parameters. Structural Equation Modeling, 14, 26-47.

McLachlan, G., \& Peel, D. (2000). Finite mixture models. New York, NY: Wiley.

Meredith, W., \& Tisak, J. (1990). Latent curve analysis. Psychometrika, $55,107-122$.

Morin, A. J. S. (2016). Person-centered research strategies in commitment research. In J. P. Meyer (Ed.), Handbook of employee commitment (pp. 490-507). Cheltenham, UK: Edward Elgar.

Morin, A. J. S., Maïano, C., Marsh, H. W., Nagengast, B., \& Janosz, M. (2013). School life and adolescents' self-esteem trajectories. Child Development, 84, 1967-1988.

Morin, A. J. S., Maïano, C., Nagengast, B., Marsh, H. W., Morizot, J., \& Janosz, M. (2011). General growth mixture analysis of adolescents' developmental trajectories of anxiety: Impact of untested invariance assumptions on interpretations. Structural Equation Modeling, 18, 613-648. doi:10.1080/10705511.2011.607714

Morin, A. J. S., Rodriguez, D., Fallu, J.-S., Maïano, C., \& Janosz, M. (2012). Academic achievement and adolescent smoking: A general growth mixture model. Addiction, 107, 819-828.

Morin, A. J. S., \& Wang, J. C.-K. (2016). A gentle introduction to mixture modeling using physical fitness data. In N. Ntoumanis \& N. Myers (Eds.), An introduction to intermediate and advanced statistical analyses for sport and exercise scientists (pp. 183-210). London, UK: Wiley.

Muthén, B. (2003). Statistical and substantive checking in growth mixture modeling: Comment on Bauer and Curran (2003). Psychological Methods, 8, 369-377. doi:10.1037/1082-989 X.8.3.369

Muthén, B. (2004). Latent variable analysis: Growth mixture modeling and related techniques for longitudinal data. In D. Kaplan (Ed.), Handbook of quantitative methodology for the social sciences (pp. 345-368). Newbury Park, CA: Sage.

Muthén, B. O., Brown, C. H., Masyn, K., Jo, B., Khoo, S. T., Yang, C. C., ... Liao, J. (2002). General growth mixture modeling for randomized preventive interventions. Biostatistics, 3, 459-475.

Muthén, B. O., \& Curran, P. J. (1997). General longitudinal modeling of individual differences in experimental designs: A latent variable framework for analysis and power estimation. Psychological Methods, 2, 371-402. doi:10.1037/1082-989X.2.4.371
Muthén, L. K., \& Muthén, B. O. (2002). How to use a Monte Carlo study to decide on sample size and determine power. Structural Equation Modeling, 9, 599-620. doi:10.1207/S15328007SEM0904_8

Muthén, L. K., \& Muthén, B. (2012). Mplus user's guide. Los Angeles, CA: Muthén \& Muthén.

Muthén, B. O., \& Shedden, K. (1999). Finite mixture modeling with mixture outcomes using the EM algorithm. Biometrics, 55, 463-469.

Nylund, K. L., Asparouhov, T., \& Muthén, B. O. (2007). Deciding on the number of classes in latent class analysis and growth mixture modeling: A Monte Carlo simulation study. Structural Equation Modeling, 14, 535-569.

Pastor, D. A., \& Gagné, P. (2013). Mean and covariance structure mixture models. In G. R. Hancock \& R. O. Mueller (Eds.), Structural equation modeling: A second course (2nd ed.). Charlotte, NC: Information Age.

Peugh, J., \& Fan, X. (2012). How well does growth mixture modeling identify heterogeneous growth trajectories? A simulation study examining GMM's performance characteristics. Structural Equation Modeling, 19, 204-226. doi:10.1080/10705511.2012.659618

Peugh, J., \& Fan, X. (2013). Modeling unobserved heterogeneity using latent profile analysis: A Monte Carlo simulation. Structural Equation Modeling, 20, 616-639.

Peugh, J., \& Fan, X. (2015). Enumeration index performance in generalized growth mixture models: A Monte Carlo test of Muthén's (2003) hypothesis. Structural Equation Modeling, 22, 115-131. doi:10.1080/10705511.2014.919823

Qureshi, I., \& Fang, Y. (2011). Socialization in open source software projects: A growth mixture modeling approach. Organizational Research Methods, 14, 208-238.

Ram, N., \& Grimm, K. J. (2009). Growth mixture modeling: A method for identifying differences in longitudinal change among unobserved groups. International Journal of Behavioral Development, 33, 565-576.

Ramaswamy, V., DeSarbo, W. S., Reibstein, D., \& Robinson, W. (1993). An empirical pooling approach for estimating marketing mix elasticities with PIMS. Marketing Science, 12, 103-124.

Redner, R. A., \& Walker, H. F. (1984). Mixture densities, maximum likelihood and the EM algorithm. SIAM Review, 26, 195-239.

Schaeffer, C. M., Petras, H., Ialongo, N., Poduska, J., \& Kellam, S. (2003). Modeling growth in boys' aggressive behavior in elementary school: Links to later criminal involvement, conduct disorder, and antisocial personality disorder. Developmental Psychology, 39, $1020-1035$.

Schwarz, G. (1978). Estimating the dimensions of a model. Annals of Statistics, 6, 461-464. doi:10.1214/aos/1176344136

Sclove, L. (1987). Application of model-selection criteria to some problems in multivariate analysis. Psychometrika, 52, 333-343.

Sterba, S. K., Prinstein, M. J., \& Cox, M. J. (2007). Trajectories of internalizing problems across childhood: Heterogeneity, external validity, and gender differences. Development and Psychopathology, 19, $345-366$.

Tofighi, D., \& Enders, C. K. (2007). Identifying the correct number of classes in growth mixture models. In G. R. Hancock \& K. M. Samuelsen (Eds.), Advances in latent variable mixture models (pp. 317-341). Charlotte, NC: Information Age.

Tolvanen, A. (2008). Latent growth mixture modeling: A simulation study (Unpiblished doctoral dissertation, University of Jyvaskyla). Retrieved from www.statmodel.com/download/rep111.pdf

Yang, C. (2006). Evaluating latent class analyses in qualitative phenotype identification. Computational Statistics and Data Analysis, 50, $1090-1104$. 